\title{
Separable Brain Systems Supporting Cued Versus Self-Initiated Realization of Delayed Intentions
}

\author{
Sam J. Gilbert \\ University College London
}

Anna-Lisa Cohen

New York University and Yeshiva University

\author{
Peter M. Gollwitzer \\ New York University and University of Konstanz \\ Gabriele Oettingen \\ New York University and University of Hamburg
}

\author{
Paul W. Burgess \\ University College London
}

\begin{abstract}
In everyday life, one can link anticipated specific cues (e.g. visiting a restaurant) with desired actions (e.g., ordering a healthy meal). Alternatively, intentions such as "I intend to eat more healthily" present the option to act when one encounters the same cue. In the first case, a specific cue triggers a specific action; in the second, one must act in a more self-initiated manner. The authors compared such scenarios using functional magnetic resonance imaging. Participants were either instructed to respond in a particular manner to target events (cued condition) or told that they would score points for such responses, without being told that they were necessary (self-initiated condition). Although conditions differed only in the wording of instructions, the self-initiated condition was associated with poorer performance and greater activity in a predominantly frontoparietal network. Responses to targets in the self-initiated and cued conditions yielded greater activity in lateral and medial Brodmann area 10 , respectively. The authors suggest that these results reflect differing demands for self-initiated versus externally cued behavior following different types of instruction, in line with the distinction between goal intentions and implementation intentions proposed by P. M. Gollwitzer and colleagues.
\end{abstract}

Empirical investigations in psychology and cognitive neuroscience typically describe experimental tasks in terms of the stimuli presented and appropriate responses but do not always report the precise manner in which participants were instructed in their initial interactions with the experimenter. Yet the nature of these instructions may have strong effects on the way that participants configure themselves to perform the relevant task(s), a process that is not well understood at present (Allport, Styles, \& Hsieh, 1994; Duncan et al., 2008; Monsell, 1996; Sakai \& Passingham, 2003,

Sam J. Gilbert and Paul W. Burgess, Institute of Cognitive Neuroscience, and Department of Psychology, University College London, London, United Kingdom; Peter M. Gollwitzer, Department of Psychology, New York University, and Department of Psychology, University of Konstanz, Konstanz, Germany; Anna-Lisa Cohen, Department of Psychology, New York University, and Department of Psychology, Yeshiva University; Gabriele Oettingen, Department of Psychology, New York University, and Department of Psychology, University of Hamburg, Hamburg, Germany.

This study was supported by the Wellcome Trust (061171), Royal Society, and the Department of Psychology at New York University. We would like to thank Clayton Curtis and Elizabeth Parks-Stamm for helpful discussions concerning this work.

Correspondence concerning this article should be addressed to Sam J. Gilbert, Institute of Cognitive Neuroscience, University College London, 17 Queen Square, London, England WC1N 3AR, United Kingdom. E-mail: sam.gilbert@ucl.ac.uk
2006; Waszak, Wenke, \& Brass, 2008). Outside the laboratory, this issue has important implications. In everyday life, we often form intentions for future behavior (i.e., instruct ourselves), and the nature of these instructions can have strong effects on how successfully we realize such delayed intentions (Gollwitzer \& Sheeran, 2006). In the present study, we used a modified prospective memory paradigm to investigate the effects of task instructions on behavioral performance and underlying brain activity. We compared two conditions with different sets of instructions. However, participants were presented with the same stimuli in both conditions, and the same responses were appropriate.

Prospective memory (PM) refers to a class of situations involving realization of delayed intentions (see, e.g., Burgess, Scott, \& Frith, 2003 for a set of characteristics). In typical laboratory studies of PM, participants perform an ongoing task in which they are presented with a series of stimuli requiring some classification (e.g., words requiring a two-alternative judgment according to their length). In PM conditions, participants are provided with an additional PM instruction to respond differently either if a particular target event happens in the future (e.g., "Press a different button if an animal word is presented") or at a particular time (e.g., "Press a different button after $30 \mathrm{~s}$ of task performance"). Thus, these paradigms are sensitive to participants' ability to encode an intended future action and to act on that intention at the appropriate time (for recent studies, see Brandimonte, Einstein, \& McDaniel, 
1996; Einstein et al., 2005; Eschen et al., 2007; Kliegel, McDaniel, \& Einstein, 2008; Marsh, Hicks, \& Cook, 2005; Smith, 2003; West, Krompinger, \& Bowry, 2005). These abilities allow us to lead independent, purposeful lives (Ellis \& Freeman, 2008) and play an important role in health-related behaviors (e.g., remembering to take medication; Liu \& Park, 2004; Wilson \& Park, 2008).

While there has been debate among different authors as to precisely what situations constitute a PM task (Kliegel et al., 2008), we use the term here simply to refer to any situation in which (a) there are at least two types of task requirements, one of which applies only to a minority of trials (PM targets); (b) the instructions explaining the responses relevant to such target trials are not directly cued at the point at which they apply; and (c) if participants disregard these instructions, it would be possible to respond to PM targets in the same manner as in other types of trial, rather than treating them as special. In typical laboratory studies of PM, participants are told that they must respond in a particular way at a particular time or if a particular event occurs. However, in everyday life, there may be many situations in which we form intentions for future actions that we subsequently have the option of performing, for example, if there are multiple opportunities to act or if we form an intention without considering exactly when to act. In these situations, we must decide to act on the intention in a more self-initiated manner, rather than being cued to do so directly. In the present study, we investigated whether behavioral performance and underlying brain activity is affected if participants are presented with the option to act, versus an instruction to act when cued.

Cognitive neuroscience studies have highlighted rostral prefrontal cortex (PFC), approximating Brodmann area 10 (BA 10), as a region playing a crucial role in PM (Burgess et al., 2003; Burgess, Quayle, \& Frith, 2001; Burgess, Veitch, Costello, \& Shallice, 2000; Okuda et al., 1998, 2007; Reynolds, West, \& Braver, in press; Simons et al., 2006, as reviewed by Burgess et al., 2008; West et al., 2008). Neuroimaging investigations typically find that performance of PM tasks, compared with performance of ongoing tasks alone, elicit increased activity in lateral BA 10 and decreased activity in medial BA 10. Burgess, Simons, Dumontheil, and Gilbert (2005) and Burgess, Dumontheil, and Gilbert (2007) have accounted for these results by suggesting that lateral BA 10 plays a role in attending to internally represented information such as intentions for future action; hence the signal in this region is increased during PM performance. By contrast, medial BA 10 is proposed to play a role in attention toward perceptual information in tasks that can be performed on the basis of well-learned stimulus-response links; hence the signal in this region is increased during performance of ongoing tasks alone (see also Braver \& Bongiolatti, 2002; Christoff, Ream, Geddes, \& Gabrieli, 2003; Gilbert, Frith, \& Burgess, 2005; Gilbert, Simons, Frith, \& Burgess, 2006; Gilbert, Spengler, Simons, Frith, \& Burgess, 2006; Gilbert, Spengler, Simons, Steele, et al., 2006; Koechlin, Basso, Pietrini, Panzer, \& Grafman, 1999).

In the behavioral literature on delayed intentions, an important distinction has been made between separable cognitive processes supporting PM in different types of situation. For instance, Einstein et al. (2005; see also McDaniel \& Einstein, 2000) suggested that in some situations, prospective remembering can occur relatively automatically, as a result of direct triggering by environ- mental cues. However, in other situations, prospective remembering may depend more heavily on deliberate monitoring of one's environment for target events. Einstein et al. (2005) proposed that the extent to which these different types of processes are engaged can depend on the "degree to which participants form a good encoding between the cue and the intended action" (p. 328).

A related distinction between processes supporting goal intentions and implementation intentions has been put forward by other authors (Gollwitzer, 1993, 1999; Gollwitzer \& Brandstätter, 1997). Goal intentions have been characterized as representations of future goal states: "I intend to attain goal Z." Implementation intentions have been characterized as representations of specific environmental events and appropriate behavioral responses that may bring about a desired goal state: "If I encounter situation $\mathrm{X}$, then I will perform behavior Y." It has been suggested that encoding a specific situation-action link (e.g. "If I am in a restaurant, then I will order a low-fat meal") is more effective than encoding an overarching goal (e.g., "I intend to lose weight"; recent reviews by Cohen \& Gollwitzer, 2006; Gollwitzer \& Sheeran, 2006). This may be because an implementation intention specifies a mandatory action when a particular cue is encountered, whereas a goal intention is more reliant on self-initiated behavior. The distinction between these two types of intention is particularly relevant to the present investigation because recent studies within the domain of PM have shown that participants are more likely to enact behaviors associated with implementation intentions than goal intentions (Chasteen, Park, \& Schwarz, 2001; Cohen \& Gollwitzer, 2008; McDaniel \& Howard, 2008).

In the literature on implementation intentions, it has been proposed that once an implementation intention has been formed, goal-directed behaviors may be produced relatively automatically, because an appropriate response is cued directly by environmental events (Brandstätter, Lengfelder, \& Gollwitzer, 2001; Lengfelder \& Gollwitzer, 2001). In other words, by forming an implementation intention we may "delegate" control of goal-directed behavior to the environment (Gollwitzer, 1999). By contrast, goal intentions are proposed to rely more heavily on deliberate monitoring of the environment for opportunities to act. Putting together the results from the literature on implementation intentions with the literature on the cognitive neuroscience of PM, one prediction might be that these two types of intentions should be associated with separable activity in lateral versus medial BA 10 . If implementation intentions promote efficient goal-directed behavior because they allow appropriate responses to be cued more directly by events in one's environment, this type of intention should be preferentially associated with the functions of medial BA 10 , because this area is involved in environmentally triggered behavior (Gilbert et al., 2005, Gilbert, Simons, Frith, \& Burgress, 2006, Gilbert, Spengler, Simons, Frith, et al., 2006). However, if goal intentions require greater attention toward internally represented goals, this type of intention should be preferentially associated with lateral BA 10 , previously implicated in stimulus-independent attention (Burgess et al., 2007; Gilbert et al., 2005, Gilbert, Spengler, Simons, Frith, et al., 2006).

In the present study, we tested these predictions by administering two separate PM tasks. For each task, we prepared two sets of instructions. In one set of instructions ("cued" condition), participants were simply told to respond to PM targets. In another set of instructions ("self-initiated" condition), participants were given the 
option of responding to PM targets. The reward structure in this condition made it clear that participants should respond to PM targets, without stating that explicitly as in the cued condition. Thus, participants in this condition had to establish a more selfinitiated strategy for responding to the PM targets, compared with the cued condition. Each participant performed one task in the cued condition and the other task in the self-initiated condition, with the assignment of instructions to tasks counterbalanced between participants. In this manner, we were able to minimize transfer of instructions from one condition to the other (because they were associated with different tasks), while maximizing the power of our design by permitting within-subject comparisons between the cued and self-initiated conditions.

In the self-initiated condition, we instructed participants that their task was to score as many points as possible and that they would score 1 point for each correct ongoing response and 5 points for each correct PM response. Participants were not instructed that points scored in the experiment corresponded with any form of reward, and there were no additional task demands related to this condition. In the cued condition, we simply instructed participants to continue performing the ongoing task and, where appropriate, to make PM responses. We hypothesized that the cued condition would encourage the formation of implementation intentions, drawing participants' attention toward the link between a specific cue and a specific action, permitting efficient environmental triggering of behavior. By contrast, we hypothesized that the selfinitiated condition would encourage the formation of goal intentions, drawing participants' attention toward an overarching goal of scoring points rather than the specific stimulus-response links that would achieve this goal.

\section{Method}

\section{Participants}

There were 16 healthy right-handed participants between the ages of 19 and 36 years (mean: 22.7 years; 11 women and 5 men) drawn from the New York University (NYU) community. The study was approved by NYU's institutional review board, and all participants provided written informed consent before taking part. Participants received a payment of $\$ 37.50$ for taking part in the study.

\section{Tasks}

There were two tasks: letters and dots (Figure 1). Stimuli were projected onto a mirror in direct view of participants, who responded by pressing buttons on a response pad with their right hand. In both tasks, participants were presented with a fixation cross in the center of the screen on each trial. In the letters task, participants were additionally presented with a letter of the alphabet on either side of the fixation cross, one of which was upper case and the other of which was lower case. Participants were instructed to indicate with a button press whether the upper-case letter was on the left or right of the screen. In the dots task, participants were presented with three yellow dots on each trial, in a random configuration, either with two dots to the left of the fixation cross and one to the right, or vice versa. Participants were instructed to indicate with a button press whether two dots could

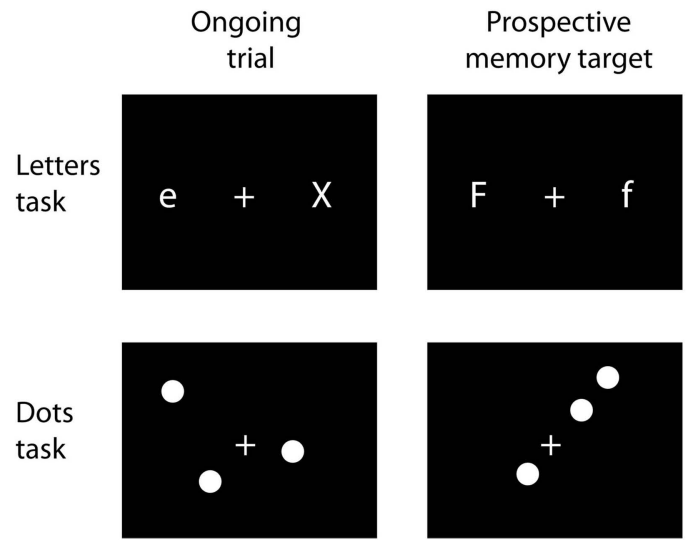

Figure 1. Schematic illustration of the two behavioral tasks. In the letters task, participants saw two letters of the alphabet (one upper-case, one lower-case) on either side of the fixation cross. The ongoing task was to indicate whether the upper-case letter was on the left or right side of the screen. In the dots task, participants saw three dots. The ongoing task was to indicate whether two dots were to the left or right side of the fixation cross. In prospective memory conditions, participants were instructed to press a third button if a target stimulus was presented, rather than producing an ongoing response. In the letters task, a target stimulus was one consisting of the same letter on both sides of the screen. In the dots task, a target stimulus was one where the three dots formed a straight line.

be seen to the left or right of the fixation cross. In the PM conditions, participants were required to press a different button whenever a PM target was presented. In the letters task, a PM target was defined as any trial in which the same letter of the alphabet was presented on both sides of the fixation cross. In the dots task, a PM target was defined as any trial in which the three dots were configured so that they all fell on a straight line.

Participants also performed a baseline task to allow comparisons to be made between sessions. In this task, participants were required to alternate between left and right key-press responses to a row of five Xs, which alternated between a horizontal and vertical orientation after each response. In the letters and dots task, the stimulus was removed from the screen immediately following each response, so only one response could be made on each trial (i.e., ongoing or PM). The next stimulus was then presented after a random pause that lasted between 150 and $350 \mathrm{~ms}$. The baseline task was also self-paced, with a response-stimulus interval chosen randomly to last between 300 and $700 \mathrm{~ms}$.

\section{Procedure}

Participants first practiced the ongoing and baseline tasks in a 5-min session prior to scanning. They then underwent two scans of approximately 4 min each, during which they performed the letters and dots ongoing tasks (in separate sessions). These sessions consisted of seven cycles of ongoing task performance (25 s) followed by baseline task performance ( $8 \mathrm{~s})$, with a 1 -s pause between each. Following these sessions, instructions relating to the PM demands of one of the tasks were presented on the computer screen. After receiving these instructions, participants practiced the task for $40 \mathrm{~s}$ and then underwent two scans of approximately 8 min. These scans consisted of 12 cycles of a task instruction 
reminder $(5 \mathrm{~s})$ followed by task performance $(25 \mathrm{~s})$ followed by baseline task performance ( $8 \mathrm{~s}$ ), with a 1 -s pause between each. Participants were then given instructions relating to the PM demands of the other task, followed by a 40 -s practice session. The participants then underwent two scans of approximately $8 \mathrm{~min}$, as described earlier. The entire scanning session lasted approximately $40 \mathrm{~min}$. The order of the two tasks, the assignment of tasks to conditions (self-initiated versus cued), and the order of conditions were fully counterbalanced between participants. Within each 25 -s block of task performance, participants were equally likely to be presented with one PM target (at a randomly selected point between the 3rd and 22nd second of the block) or two PM targets (one between the 3rd and 11 th second of the block and the other between the 14th and 22nd second).

\section{Instructions}

Participants first performed the ongoing tasks alone, before being introduced to the PM instructions, to avoid "contamination" of ongoing task performance (Simons, Schölvinck, Gilbert, Frith, $\&$ Burgess, 2006). Subsequently, PM instructions were introduced via the computer screen. Participants in the self-initiated condition received the following instructions: "In this part of the experiment, you must try to score as many points as possible." They were told that they would score 1 point for every ongoing trial and 5 points if they pressed a PM response key (the middle button on the keypad) when a target was presented. They were then asked silently to read an instruction phrase that was presented three times (e.g., in the letters task, "IF the same letter is on both sides, THEN I can score 5 points!"). In the cued condition, participants were simply asked to continue performing the ongoing task and told that if a target was presented, they must press the PM response key. The instruction phrase used in this condition for the letters task was "IF the same letter is on both sides, THEN I will press the middle button!" Equivalent instructions were prepared for the dots task. These instruction phrases were then repeated throughout task performance as reminders. The self-initiated and cued conditions therefore differed only in the wording of instructions given to participants on the computer screen. In the self-initiated condition, a link was made between a cue and a particular goal without the participants receiving explicitly instructions on how to respond, whereas in the cued condition, a direct link was made between a cue and a particular response, as in previous investigations of implementation intentions (Gollwitzer \& Sheeran, 2006).

\section{Imaging Methods}

A 3-Tesla Siemens Allegra head-only system (Siemens Inc., Erlangen, Germany) was used to acquire T2*-weighted echoplanar images (EPIs; $64 \mathrm{~mm} \times 64 \mathrm{~mm} ; 3 \mathrm{~mm} \times 3 \mathrm{~mm}$ pixels; echo time [TE], $30 \mathrm{~ms}$ ) with blood-oxygen-level-dependent (BOLD) contrast. Each volume comprised 36 axial slices (2-mm thick, separated by $1.7 \mathrm{~mm}$ ), oriented at approximately $10^{\circ}$ to the anterior commissure-posterior commissure plane, covering the whole brain. Functional magnetic resonance images (fMRI) were acquired during six sessions, the first two of which comprised 110 volumes and the last four of which comprised 216 volumes. Volumes were acquired continuously with an effective repetition time (TR) of $2.34 \mathrm{~s}$ per volume. The first five volumes in each session were discarded to allow for T1 equilibration effects.

\section{Data Analysis}

MRI data were analyzed with SPM5 software (available at http://www.fil.ion.ucl.ac.uk/spm/spm5.html). Volumes were realigned, corrected for different slice acquisition times, normalized into 2-mm-cubic voxels using a standard EPI template based on the Montreal Neurological Institute reference brain using fourthdegree B-spline interpolation, and smoothed with an isotropic 8-mm full-width half-maximum Gaussian kernel. The volumes acquired during the six sessions were treated as separate time series. For each series, the variance in the BOLD signal was decomposed with a set of regressors in a general linear model (Friston et al., 1995). In the first two sessions (ongoing only), variance was decomposed into components associated with sustained activity during ongoing task performance, as well as sustained activity during performance of the baseline task. In the subsequent four sessions, additional regressors coded for sustained activity during instruction presentation, along with two regressors representing transient activity elicited by correctly detected PM targets and missed PM targets. Sustained activity was modeled with boxcar regressors, convolved with a canonical hemodynamic response function (HRF); transient activity was modeled with delta functions, convolved with a canonical HRF. The full model for each session comprised these regressors, together with regressors representing residual movement-related artefacts and the mean over scans. The data and model were high-pass filtered to a cutoff of $1 / 128 \mathrm{~Hz}$.

Parametric estimates for each regressor were calculated from the least mean squares fit of the model to the data. Results were assessed in random effects analyses with one-sample $t$ tests on contrast images conducted at the subject-specific level. Because participants cannot be expected to "unlearn" PM instructions after they have been exposed to them (Simons et al., 2006), we had to make comparisons between conditions administered in different scanning sessions. We achieved this by first comparing the signal associated with each experimental condition with the baseline condition within the relevant session, as in the previous study by Simons et al. (2006). For consistency with this study, contrasts were thresholded at $p<.001$, uncorrected for multiple comparisons, with a minimum extent of five contiguous voxels. Given that this is an uncorrected threshold, activations outside the a priori regions of interest (medial and lateral BA 10) should be interpreted with caution.

\section{Results}

\section{Behavioral Data}

In the following analyses, results were collapsed over the dots and letters task, so that within-subject comparisons could be made between self-initiated and cued conditions. This was made possible by strict counterbalancing of the assignment of the two conditions to the two tasks and the order of the two conditions, such that the distinction between self-initiated and cued conditions was not confounded with any other factor at the group level. During PM blocks, the mean proportion of trials on which a target was 
presented was $5.6 \%$. By comparing reaction times (RTs) to the ongoing task (e.g., letter case discrimination) between the initial "uncontaminated" blocks and subsequent PM blocks, we were able to assess the "cost" to RTs of bearing in mind the PM instructions (Einstein et al., 2005; Smith, 2003). In both the self-initiated and cued conditions, RTs were slower in the PM blocks than in the initial uncontaminated ongoing blocks-self-initiated: $t(15)=7.1$, $p<.001$; cued: $t(15)=5.1, p<.001$ (see Figure 2). However, the cost associated with PM blocks did not differ significantly between the self-initiated and cued conditions, $F(1,15)=1.3, p=.26$. Mean error rates were low $(<2 \%)$ and did not differ significantly between conditions $(F<1)$. Within PM blocks, analysis of the percentage of targets receiving a PM response rather than an ongoing response revealed that participants were more likely to respond correctly to PM targets in the cued condition (75.7\%) than in the self-initiated condition $(65.0 \%), t(15)=3.6, p=.003$; Figure 2. Calculation of the effect size for this comparison indicated that this represents a large effect (Cohen's $d=0.9$ ).

\section{Neuroimaging Data}

When collapsed across cued and self-initiated conditions, the contrast between sustained activity during task performance in the PM blocks and task performance in the uncontaminated ongoing blocks revealed greater activity in medial rostral PFC during the ongoing blocks $\left(2,56,-2 ; \mathrm{BA} 10 ; \mathrm{Z}_{\max }=4.84 ; 1511\right.$ voxels $)$, as expected on the basis of previous studies (Burgess et al., 2003; Simons et al., 2006). This effect was also found individually in both the cued and self-initiated conditions (cued: 8, 62, -6; BA $10 ; \mathrm{Z}_{\max }=3.48 ; 10$ voxels; self-initiated: $-4,48,-8$; BA 10 ; $\mathrm{Z}_{\max }=4.50 ; 620$ voxels $)$. However, of more interest in the present study was the direct comparison of cued and self-initiated conditions within the PM blocks.

First, we investigated brain regions that differed in activity during ongoing task performance in the two conditions (i.e., sustained differences in activity between the two conditions, rather than activity linked to PM targets). There were no brain regions showing significantly greater activity in the cued condition. In the self-initiated condition, significantly greater activity was seen in left ventrolateral PFC, left insula, bilateral supplementary motor area, bilateral posterior cinguate/precuneus, left inferior and superior parietal cortex, right lateral temporal cortex, and left medial temporal lobe (Table 1; Figure 3, left panel).

Next, we directly compared transient activity associated with correctly detected PM targets in the two conditions (Table 2; Figure 3, right panel). In the self-initiated condition (selfinitiated $>$ cued), greater activity was seen in bilateral lateral rostral PFC (BA 10), along with more posterior lateral frontal regions, insula, bilateral ventral temporal cortex, right inferior parietal cortex and left lateral occipital cortex. In the reverse contrast (cued > self-initiated), greater activity was seen in medial rostral PFC (BA 10), left premotor cortex, superior temporal cortex, right insula, and right lateral occipital cortex. These results confirmed the predicted lateral versus medial dissociation within BA 10 . We further investigated these data by examining signal at the peak co-ordinates previously reported in the study by Simons et al. (2006). This analysis allowed us to avoid the problem of multiple comparisons, permitting strong conclusions to be drawn using an uncorrected statistical threshold. In the earlier study, the contrast between PM and ongoing conditions produced three activation peaks in BA 10, all in lateral regions: $-39,57,3 ; 42,57$, 9 ; and $39,54,15$. The present contrast between self-initiated and

\section{\% PM targets detected}

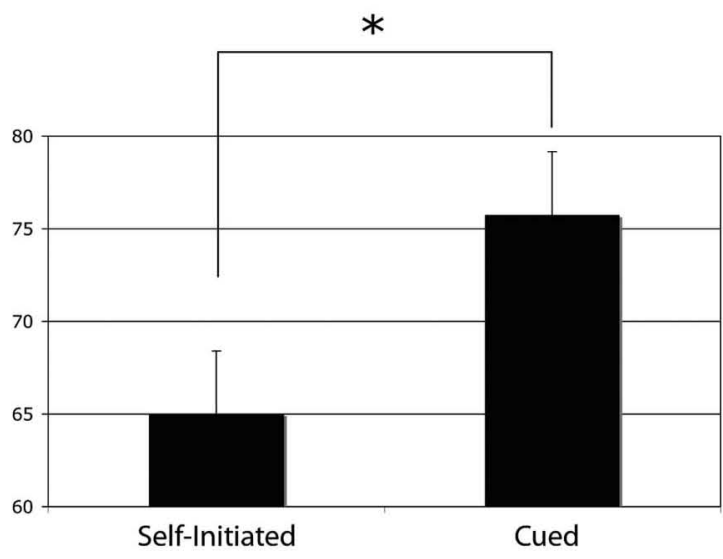

\section{Mean RT to ongoing trials}

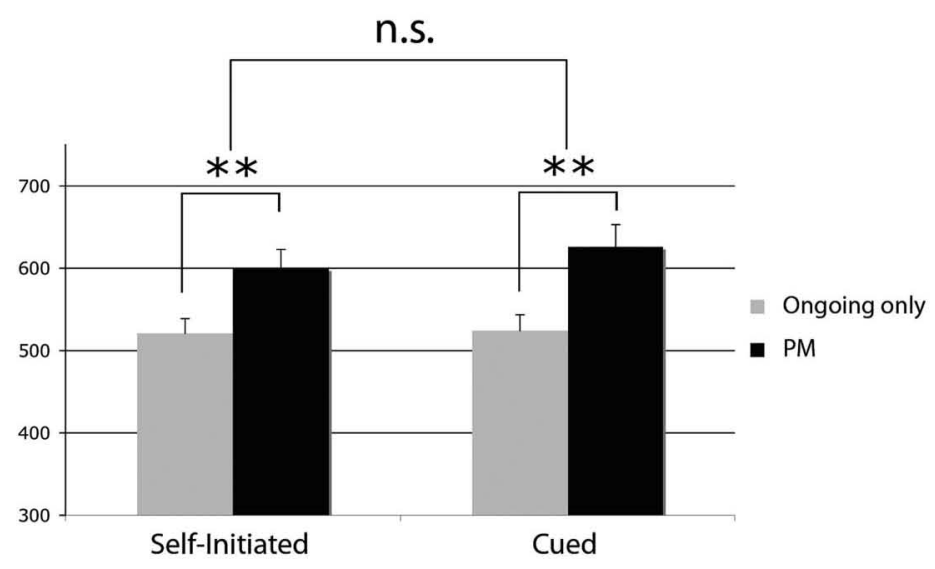

$$
\begin{aligned}
* \mathrm{p} & <.005 \\
* * \mathrm{p} & <.0002
\end{aligned}
$$

Figure 2. Behavioral data. Left panel: Mean percentage of prospective memory (PM) targets detected in the self-initiated and cued conditions. Right panel: Mean reaction time (RT) in the ongoing tasks. Pale gray bars indicate RTs during the ongoing task at the beginning of the experiment, before prospective memory (PM) instructions were introduced. Black bars indicate reaction times after PM instructions were introduced. Error bars indicate standard error of the mean. 
Table 1

Regions Exhibiting Significantly Greater Sustained Activity During Performance of the Self-Initiated Than During the Performance of the Cued Condition (i.e., Throughout Ongoing Performance)

\begin{tabular}{|c|c|c|c|c|c|c|c|}
\hline Region & $\mathrm{BA}$ & Hemisphere & $x$ & $y$ & $z$ & $Z_{\max }$ & $N$ voxels \\
\hline Ventrolateral prefrontal cortex & 47 & $\mathrm{~L}$ & -38 & 36 & -10 & 3.65 & 7 \\
\hline Insula & 13 & $\mathrm{~L}$ & -44 & 2 & 0 & 3.15 & 5 \\
\hline \multirow[t]{2}{*}{ Supplementary motor area } & 6 & $\mathrm{~L}$ & -10 & 0 & 60 & 3.48 & 7 \\
\hline & 6 & $\mathrm{R}$ & 14 & -4 & 60 & 3.38 & 6 \\
\hline Insula & 13 & $\mathrm{~L}$ & -42 & -10 & 0 & 3.28 & 7 \\
\hline \multirow[t]{2}{*}{ Posterior cingulate } & 24 & $\mathrm{R}$ & 18 & -16 & 44 & 3.75 & 18 \\
\hline & 31 & $\mathrm{~L}$ & -10 & -32 & 38 & 3.36 & 11 \\
\hline Inferior parietal cortex & 40 & $\mathrm{~L}$ & -58 & -38 & 40 & 3.95 & 29 \\
\hline Posterior cingulate & 31 & $\mathrm{R}$ & 16 & -38 & 46 & 3.48 & 5 \\
\hline Lateral temporal cortex & 22 & $\mathrm{R}$ & 66 & -44 & 18 & 3.55 & 6 \\
\hline Precuneus & 7 & $\mathrm{R}$ & 6 & -44 & 58 & 3.35 & 6 \\
\hline Medial temporal lobe & 19 & $\mathrm{~L}$ & -24 & -46 & -8 & 3.36 & 5 \\
\hline Superior parietal cortex & 7 & $\mathrm{~L}$ & -24 & -48 & 66 & 3.32 & 7 \\
\hline
\end{tabular}

Note. Co-ordinates refer to the Montreal Neurological Institute reference brain. Brodmann areas $(\mathrm{BA})$ are approximate. $\mathrm{L}=1 \mathrm{eft}$; $=$ right.

cued conditions revealed significant effects in all three regions, $t(15)>2.2, p<.02$. Simons et al. also found that the contrast between ongoing and PM conditions produced activation in medial BA 10: $0,48,-6$. In this region, the present contrast between self-initiated and cued conditions also revealed an effect that just missed significance, $t(15)=1.6, p<.07$.

To investigate potential links between individual differences in brain activity between the two conditions and behavioral differences, we investigated correlations between (a) the difference in the percentage of PM targets detected in the self-initiated versus cued condition, and (b) the difference in activity elicited by correctly-detected targets in the two conditions. Left lateral rostral PFC (-34, 54, 0; BA 10) was unique among the regions identified earlier in showing a significant correlation between these two

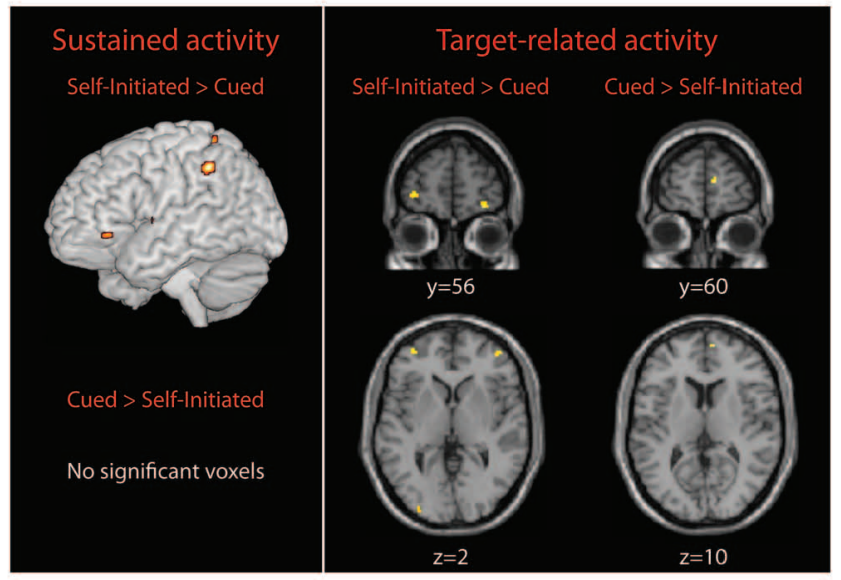

Figure 3. Left panel: Three-dimensional rendering of brain regions showing differences in activity during ongoing trials in the comparison between self-initiated and cued conditions. Differences in activity can be seen in left frontal and parietal regions. Right panel: Brain regions showing differences in target-related activity between the self-initiated and cued conditions, plotted on coronal and axial slices of a normalized T1-weighted scan. These results indicate opposite effects in lateral versus medial rostral prefrontal cortex. measures $(r=-0.73, p=.0001$; all other regions: $|r|<.3, p\rangle$ .26). Participants who showed the greatest difference in activity in this region between the two conditions (with more activity in the self-initiated than the cued condition) also tended to show the greatest behavioral difference (with fewer targets detected in the selfinitiated than the cued condition). In other words, to the extent that participants activated this region more in the self-initiated condition (relative to the cued condition), they also detected fewer targets in this condition.

\section{Discussion}

In this study, we contrasted brain activity supporting realization of delayed intentions in two conditions that differed only in the pretask instructions provided to participants. In the cued condition, participants were asked to produce a specific response whenever a target event occurred. In the self-initiated condition, participants were given the option of doing so, although the reward structure made it clear that they should respond to targets as in the cued condition. There were four main findings: (a) The cued condition produced superior PM performance to the self-initiated condition. (b) Sustained throughout task performance, the self-initiated condition was associated with activation in a predominantly frontoparietal network (i.e., greater activation in the condition with poorer performance), whereas the cued condition was not associated with significant activation in any region. (c) The two conditions were associated with differential patterns of activation in rostral PFC (BA 10): Responding to PM targets in the self-initiated condition was associated with greater activation in bilateral lateral $\mathrm{BA} 10$, whereas responding to PM targets in the cued condition was associated with greater activation in medial BA 10. (d) The difference in target-related activity between these two conditions in left lateral BA 10 mirrored the behavioral difference between the conditions, with greater activity associated with poorer performance. This result was not obtained in any of the other regions activated by the contrasts between the two conditions.

These results show that the way that participants are instructed to perform a task can have significant effects on both behavior and underlying brain activity. This finding underlines the importance 
Table 2

Significant Differences in Activity Transiently Associated With Correctly Detected Prospective Memory Targets in the Self-Initiated and Cued Conditions

\begin{tabular}{|c|c|c|c|c|c|c|c|}
\hline Region & $\mathrm{BA}$ & Hemisphere & $x$ & $y$ & $z$ & $Z_{\max }$ & $N$ voxels \\
\hline & \multicolumn{7}{|c|}{ Self-initiated $>$ cued } \\
\hline \multirow[t]{5}{*}{ Lateral prefrontal cortex } & $10 / 11$ & $\mathrm{R}$ & 30 & 56 & -12 & 3.75 & 26 \\
\hline & 10 & $\mathrm{~L}$ & -34 & 54 & 0 & 4.15 & 44 \\
\hline & 10 & $\mathrm{R}$ & 46 & 54 & 4 & 3.69 & 28 \\
\hline & 47 & $\mathrm{~L}$ & -34 & 26 & -12 & 3.74 & 11 \\
\hline & $45 / 46$ & $\mathrm{~L}$ & -38 & 26 & 22 & 3.65 & 9 \\
\hline Insula & 47 & $\mathrm{R}$ & 24 & 22 & -6 & 3.29 & 5 \\
\hline Superior frontal cortex & 8 & $\mathrm{~L}$ & -24 & 16 & 44 & 4.07 & 17 \\
\hline \multirow[t]{2}{*}{ Ventral temporal cortex } & 20 & $\mathrm{R}$ & 46 & -2 & -38 & 3.78 & 14 \\
\hline & 20 & $\mathrm{~L}$ & -44 & -12 & -32 & 3.45 & 5 \\
\hline Premotor cortex & 6 & $\mathrm{~L}$ & -32 & -14 & 66 & 3.64 & 5 \\
\hline Insula & - & $\mathrm{L}$ & -26 & -22 & 14 & 3.77 & 12 \\
\hline \multirow[t]{2}{*}{ Inferior parietal cortex } & 40 & $\mathrm{R}$ & 52 & -48 & 52 & 3.85 & 58 \\
\hline & 40 & $\mathrm{R}$ & 58 & -48 & 40 & 3.39 & 19 \\
\hline \multirow[t]{2}{*}{ Lateral occipital cortex } & 18 & $\mathrm{~L}$ & -30 & -92 & -2 & 3.61 & 21 \\
\hline & \multicolumn{7}{|c|}{ Cued $>$ self-initiated } \\
\hline Medial rostral prefrontal cortex & 10 & $\mathrm{R}$ & 10 & 60 & 10 & 3.61 & 5 \\
\hline \multirow[t]{2}{*}{ Premotor cortex } & 6 & $\mathrm{~L}$ & -20 & 2 & 60 & 3.32 & 6 \\
\hline & 6 & $\mathrm{~L}$ & -22 & -2 & 42 & 3.55 & 5 \\
\hline Superior temporal cortex & 41 & $\mathrm{~L}$ & -52 & -20 & 12 & 3.82 & 6 \\
\hline Insula & - & $\mathrm{R}$ & 26 & -30 & 18 & 3.92 & 5 \\
\hline Lateral occipital cortex & 37 & $\mathrm{R}$ & 40 & -66 & 6 & 3.39 & 5 \\
\hline
\end{tabular}

Note. Co-ordinates refer to the Montreal Neurological Institute reference brain. Brodmann areas (BA) are approximate. $\mathrm{L}=$ left; $\mathrm{R}=$ right.

of standardizing instructions in order to compare results across studies and the importance of reporting not only the tasks that participants performed, but also the way in which a task was introduced to participants. It is also of relevance to studies comparing two or more populations (e.g. particular clinical groups versus control participants, or younger versus older adults) who may have received different instructions prior to testing. In an analogous manner to studies of retrospective memory, which have demonstrated strong effects of the encoding task on subsequent memory (e.g. Craik \& Tulving, 1975), the present results indicate that changes in the way in which participants encode prospective memory instructions can have strong effects on behavior and underlying brain activity (see also Nowinski \& Dismukes, 2005; Paul et al., 2007).

In the following sections, we consider the relationship between these results and three potential ways in which the self-initiated and cued conditions may have differed in the present study: (a) differences between the conditions in rewardrelated processing, (b) differences between the conditions in the relative priority given to $\mathrm{PM}$ versus ongoing components of the tasks, and (c) differences between the conditions in the degree to which behavior was self-initiated rather than being directly triggered by environmental events. We then consider the relationship between these results and the distinction between goal intentions and implementation intentions (Gollwitzer, 1999). Finally, we discuss the issue of sustained versus transient processes contributing to PM and suggest a framework for understanding the present results in terms of a continuum between self-initiated and environmentally cued behavior.

\section{Reward-Related Processing}

One potential account of the neuroimaging findings would be that the regions showing increased activity in the self-initiated condition were involved in reward-related processing during this condition, because participants were instructed to earn points. However, participants were not instructed that points scored in this condition corresponded in any way with an external reward (e.g., money). Moreover, the brain regions activated in this condition (predominantly lateral PFC and parietal regions) were quite different from those typically implicated in reward-related processing, such as the striatum and orbitofrontal cortex (Elliott, Newman, Longe, \& Deakin, 2003).

\section{Importance of PM Versus Ongoing Task Components}

Turning now to potential differences in the relative priority of PM versus ongoing components of the tasks, it is important to note that participants in the self-initiated condition were instructed that they "must score as many points as possible" and that correctly responding to PM targets was worth five times as many points as a correct ongoing response. Thus, in both self-initiated and cued conditions, a failure to respond correctly to a PM target was a failure to follow the task instructions. However, might it be possible that the cued condition encouraged participants to give greater priority or importance to the PM component of the tasks than the self-initiated condition, leading to superior performance?

We cannot rule out such a possibility. However, differential prioritization of the PM instructions in the two conditions cannot provide a full account of the present findings. Although the cued 
condition was associated with superior behavioral performance, this condition was associated with reduced brain activation during task performance. Moreover, this condition was associated with targetrelated activity in medial BA 10 (previously implicated in tasks with low cognitive demand), whereas the self-initiated condition was associated with activation in lateral BA 10 (previously implicated in tasks with high cognitive demand; Gilbert, Spengler, Simons, Frith, et al, 2006). Thus, in order to argue that the cued condition was associated with better performance because of greater PM-related "effort" in this condition, it would be necessary to argue that this increase in effort was accompanied by a reduction in brain activation. Alternatively, it might be argued that greater effort was expended on the PM component of the tasks in the self-initiated condition, because in this condition participants are explicitly told that correct responses to PM targets are worth more points than responses to ongoing trials. However, this hypothesis is not consistent with the finding that behavioral performance was reduced in this condition, whereas previous studies in which the importance of PM demands have been manipulated have generally found that participants' PM accuracy increases, and is certainly not reduced, when they are told that this aspect of the task is more important (Einstein et al., 2005; Kliegel, Martin, McDaniel, \& Einstein, 2001). It therefore seems that even if participants attributed different priority to the PM component of the tasks in the two conditions, this cannot explain the relationship between behavioral and neuroimaging results.

\section{Environmental Cueing and Implementation Intentions}

We now turn to potential differences in environmental cuing between the two conditions. Previous studies have implicated medial BA 10 in environmentally driven behavior, and lateral BA 10 in tasks requiring attention to be diverted toward self-generated information (Burgess et al., 2003; Gilbert, Frith, \& Burgess, 2005, Gilbert, Simons, Frith, \& Burgress, 2006, Gilbert, Spengler, Simons, Frith, et al., 2006; Gilbert et al., 2007). For example, in a study by Gilbert et al. (2005), participants performed three separate tasks that could be accomplished either by attending to visually presented information or by performing the same task "in their heads." In all three tasks, medial BA 10 activity was associated with phases of the tasks in which participants attended to visually presented information, whereas lateral BA 10 activity was associated with the process of switching attention between perceptual and self-generated information. Consistent with this suggestion, evidence from task switching suggests that lateral BA 10 is particularly engaged when participants themselves infer which of two tasks to perform, rather than being directly cued (Forstmann, Brass, Koch, \& von Cramon, 2005; see also Forstmann et al., 2008). Applying these results to the present findings, this suggests that participants were able to perform in the cued condition in a more environmentally driven manner, whereas performance in the self-initiated condition was more dependent on attention toward internally represented information.

The present results provide a link between the behavioral literature on implementation intentions and the cognitive neuroscience of prospective memory. In the behavioral literature, it has been suggested that forming an implementation intention (i.e., an ifthen plan) allows a goal-directed response to be triggered relatively automatically by an appropriate cue in an externally driven manner (Gollwitzer, 1993). In this way, implementation intentions are thought to represent an efficient mechanism for goal attainment. By contrast, goal intentions (i.e., representations of overarching goals rather than specific cue-action links) may be less effective because they are more dependent on self-initiated behavior (Cohen, Bayer, Jaudas, \& Gollwitzer, 2008; Gollwitzer \& Sheeran, 2006; see also Achtziger, Gollwitzer, \& Sheeran, 2008, for evidence that implementation intentions may in addition be triggered by internally generated cues such as craving for chocolate). In the cognitive neuroscience of prospective memory, it has been suggested that rostral prefrontal cortex (approximating BA 10) plays a crucial role due to the requirement to flexibly mediate the attentional balance between internally represented information (such as delayed intentions) and externally derived perceptual information relevant to the ongoing task (Burgess et al., 2008). Several studies have suggested that externally triggered behavior is particularly associated with the functions of medial BA 10, and self-initiated behavior is particularly associated with the functions of lateral BA 10 (Burgess et al., 2007; Gilbert, Frith, \& Burgess., 2005, Gilbert, Simons, Frith, \& Burgress, 2006; Gilbert, Spengler, Simons, Frith, et al., 2006). Bringing these two perspectives together, the present results suggest that realizing implementation intentions may depend particularly on externally cued processing, supported by medial BA 10 , whereas realizing goal intentions may depend more on self-generated processing, supported by lateral BA 10 .

\section{Sustained Versus Transient Processes}

One unresolved issue concerns the role of lateral BA 10 in item-specific "checking" (Guynn, 2003) versus sustained processes related to PM demands. While some studies implicate BA 10 in sustained processing, unrelated to specific items (Burgess et al., 2001; Reynolds, West, \& Braver, in press), the present study found transient differences in BA 10 activity between the cued and self-initiated conditions at the point of target trials, rather than sustained throughout task performance. This would be consistent with Gilbert et al.'s (2005) finding of transient lateral BA 10 activity at the point of a switch in attention between perceptually derived and self-generated information. There is therefore evidence for a role of BA 10 in both sustained and transient itemrelated processes in PM paradigms.

In the present study, sustained activity in medial BA 10 was greater during performance of the ongoing tasks alone than during PM conditions. This replicates the findings of previous neuroimaging studies investigating PM (Burgess et al., 2003; Simons et al., 2006), consistent with a role of medial BA 10 in situations in which responses can be based on externally derived information (e.g., well-learned ongoing tasks) rather than on attention to internally represented information such as representations of delayed intentions (Burgess et al., 2008; Gilbert et al., 2005). However, unlike these earlier studies, no significant difference was observed in lateral BA 10. This may reflect relatively low power in the present study to detect differences between PM and ongoing-only conditions, because the ongoing-only conditions were only performed for a relatively short time in order to maximize power in the direct comparisons between self-initiated and cued conditions.

The main focus of the present study was the contrast within PM blocks between self-initiated and cued conditions. This revealed 
increased activation in a predominantly frontoparietal network during the self-initiated condition compared with the cued condition. Similar regions of activation in ventrolateral PFC and lateral parietal cortex have been reported in several studies requiring maintenance of mnemonic information over a delay (i.e., working memory; Owen et al., 1999). More generally, Owen (2006) has argued that ventrolateral PFC is "important for intended action, that is, any behavior (e.g., an action or a thought) that is consciously willed by the agent responsible for carrying out that behavior" (p. 329). In addition, regions of left partietal cortex similar to the area activated in the present study have been suggested to represent motor intentions (Hesse, Theil, Stephan, \& Fink, 2006). Thus, the pattern of activation during the self-initiated condition may reflect the demand for active maintenance of PM instructions (e.g., rehearsal in working memory). Further studies will be required to distinguish the various forms of self-initiated processing potentially contributing to the present tasks (e.g., rehearsal of task instructions vs. monitoring for target events). Perhaps surprisingly, the cued condition, which was associated with superior performance, was not associated with significantly increased activity in any brain region. However, these results are consistent with the hypothesis that the cued condition allowed more efficient triggering of behavior by environmental cues than the self-initiated condition (cf. Gollwitzer, 1993).

\section{Continuum Between Self-Initiated and Environmentally Cued Behavior}

To the extent that participants activated lateral BA 10 to a greater degree when they detected targets in the self-initiated rather than in the cued condition, they detected fewer targets in this condition. This brain-behavior correlation was not observed in any of the other regions activated by the comparison between the two conditions. This suggests that performing the present tasks in a more self-initiated manner is a less efficient strategy for prospective remembering than encoding a more direct link between an external cue and a particular response (associated with greater medial BA 10 activity). To the extent that some participants utilized the former strategy in the self-initiated condition, this may have led to increased lateral BA 10 activity and reduced behavioral performance, compared with the cued condition, explaining the somewhat counterintuitive association between increased lateral BA 10 activity and reduced behavioral performance. However, this is not to say that participants exclusively used one strategy or the other. It may be more plausible that prospective remembering in all conditions was associated with a combination of self-initiated monitoring (supported by lateral BA 10) and externally cued behavior (supported by medial BA 10).

Both the behavioral and neuroimaging data support this conclusion. In the behavioral data, the self-initiated and cued conditions were associated with a comparable PM RT cost, suggesting a considerable degree of self-initiated monitoring even in the cued condition (Einstein et al., 2005; Smith, 2003). Conversely, it is likely that the self-initiated condition involved a considerable degree of externally cued processing, since participants were repeatedly reminded of the appearance of target stimuli in the instructions. However, given that the number of targets detected was greater in the cued than in the self-initiated condition, with no additional RT cost, this suggests that direct environmental cueing of PM was more efficient in the former condition (Figure 2; see McNerney \& West, 2007, for further evidence that the same RT cost can "buy" different levels of PM accuracy, depending on the experimental conditions). In the neuroimaging data, when the cued and self-initiated conditions were examined separately, both were associated with signal change in medial BA 10 for the comparison between ongoing and PM blocks, again suggesting the occurrence of self-initiated processing in both cued and self-initiated conditions (Burgess et al., 2003). However, direct comparison of the two indicated greater activity in medial BA 10 for the cued than the self-initiated condition. The behavioral and neuroimaging data therefore converge to suggest (a) that both the cued and the self-initiated conditions were associated with the occurrence of self-initiated processing, relative to the ongoing conditions and (b) that the degree of PM-related self-initiated processing differed between the two, being greater in the self-initiated than in the cued condition. We propose that different PM tasks will fall along a continuum depending on the extent to which they can be performed in an environmentally cued manner rather than depending on self-initiated processing, much as other tasks have been described as falling on a continuum between automatic and controlled processing (Cohen, Dunbar \& McClelland, 1990; Dunbar \& MacLeod, 1984). The present results suggest that different forms of task instruction may shift the position of PM tasks on this continuum.

In conclusion, the present results lend further support to the view that prospective memory depends on multiple, potentially dissociable cognitive processes (e.g. McDaniel \& Einstein, 2000; Simons et al., 2006). Our findings suggest that PM performance is strongly influenced by the way in which instructions are worded. More specifically, these results provide neuroimaging evidence supporting the hypothesis that an important determinant of the success of prospective remembering is the degree to which participants are able to encode intentions in a manner that allows direct environmental triggering of behavior (Gollwitzer, 1993). Spelling out a mandatory cue-action link leads to more efficient prospective memory and distinct brain activation, compared with a condition in which participants are given the option of implementing such a link in a more self-initiated manner.

\section{References}

Achtziger, A., Gollwitzer, P. M., \& Sheeran, P. (2008). Implementation intentions and shielding goal striving from unwanted thoughts and feelings. Personality and Social Psychology Bulletin, 34, 381-393.

Allport, D. A., Styles, E. A., \& Hsieh, S. (1994). Shifting intentional set: Exploring the dynamic control of tasks. In C. Umilta \& M. Moscovitch (Eds.), Attention and performance XV: Conscious and unconscious information processing (pp. 421-452). Cambridge, MA: MIT Press.

Brandimonte, G., Einstein, G., \& McDaniel, M. (1996). Prospective memory: Theory and applications. Hillsdale, NJ: Erlbaum.

Brandstätter, V., Lengfelder, A., \& Gollwitzer, P. M. (2001). Implementation intentions, and efficient action initiation. Journal of Personality and Social Psychology, 81, 946-960.

Braver, T. S., \& Bongiolatti, S. R. (2002). The role of frontopolar cortex in subgoal processing during working memory. Neurolmage, 15, 523-536.

Burgess, P. W., Dumontheil, I., \& Gilbert, S. J. (2007). The gateway hypothesis of rostral PFC (area 10) function. Trends in Cognitive Sciences, 11, 290-298.

Burgess, P. W., Dumontheil, I., Gilbert, S. J., Okuda, J., Schölvinck, M. L., \& Simons, J. S. (2008). On the role of rostral prefrontal cortex (area 10) 
in prospective memory. In M. Kliegel, M. A. McDaniel, \& G. O. Einstein (Eds.), Prospective memory: Cognitive, neuroscience, developmental, and applied perspectives (pp. 235-260). Mahwah, NJ: Erlbaum.

Burgess, P. W., Quayle, A., \& Frith, C. D. (2001). Brain regions involved in prospective memory as determined by positron emission tomography. Neuropsychologia, 39, 545-555.

Burgess, P. W., Scott, S. K., \& Frith, C. D. (2003). The role of the rostral frontal cortex (area 10) in prospective memory: A lateral versus medial dissociation. Neuropsychologia, 41, 906-918.

Burgess, P. W., Simons, J. S., Dumontheil, I., \& Gilbert, S. J. (2005). The gateway hypothesis of rostral PFC function. In J. Duncan, L. Phillips, \& P. McLeod (Eds.) Measuring the mind: Speed control and age (pp. 215-246). Oxford, UK: Oxford University Press.

Burgess, P. W., Veitch, E., Costello, A., \& Shallice, T. (2000). The cognitive and neuroanatomical correlates of multitasking. Neuropsychologia, 38, 848-863.

Chasteen, A. L., Park, D. C., \& Schwarz, N. (2001). Implementation intentions and facilitation of prospective memory. Psychological Science, $12,457-461$

Christoff, K., Ream, J. M., Geddes, L. P. T., \& Gabrieli, J. D. E. (2003). Evaluating self-generated information: Anterior prefrontal contributions to human cognition. Behavioral Neuroscience, 117, 1161-1168.

Cohen, A-L., Bayer, U. C., Jaudas, A., \& Gollwitzer, P. M. (2008). Self-regulatory strategy and executive control: Implementation intentions modulate task switching and Simon task performance. Psychological Research, 72, 12-26.

Cohen, A.-L., \& Gollwitzer, P. M. (2006). If-then plans and the intentional control of thoughts, feelings, and actions. In N. Sebanz \& W. Prinz (Eds.), Disorders of volition (pp. 151-171). Cambridge, MA: MIT Press.

Cohen, A.-L., \& Gollwitzer, P. M. (2008). The cost of remembering to remember: Cognitive load and implementation intentions influence ongoing task performance. In M. Kliegel, M. A. McDaniel, \& G. O. Einstein (Eds.), Prospective memory: Cognitive, neuroscience, developmental, and applied perspectives (pp. 367-390). Mahwah, NJ: Erlbaum.

Cohen, J. D., Dunbar, K., \& McClelland, J. L. (1990). On the control of automatic processes: A parallel distributed processing account of the Stroop effect. Psychological Review, 97, 332-361.

Craik, F. I. M., \& Tulving, E. (1975). Depth of processing and the retention of words in episodic memory. Journal of Experimental Psychology: General, 104, 268-294.

Dunbar, K., \& MacLeod, C. (1984). A horse race of a different color: Stroop interference patterns with transformed words. Journal of Experimental Psychology: Human Perception and Performance, 8, 875-894.

Duncan, J., Parr, A., Woolgar, A., Thompson, R., Bright, P., Cox, S., et al. (2008). Goal neglect and Spearman's g: Competing parts of a complex task. Journal of Experimental Psychology: General, 137, 131-148.

Einstein, G. O., McDaniel, M. A., Thomas, R., Mayfield, S., Shank, H., Morrisette, N., \& Breneiser, J. (2005). Multiple processes in prospective memory retrieval: Factors determining monitoring versus spontaneous retrieval. Journal of Experimental Psychology: General, 134, 327-342.

Elliott, R., Newman, J. L., Longe, O. A., \& Deakin, J. F. (2003). Differential response patterns in the striatum and orbitofrontal cortex to financial reward in humans: A parametric functional magnetic resonance imaging study. Joumal of Neuroscience, 23, 303-307.

Ellis, J. A., \& Freeman, J. E. (2008). Ten years on: Realizing delayed intentions. In M. Kliegel, M. A. McDaniel, \& G. O. Einstein (Eds.), Prospective memory: Cognitive, neuroscience, developmental, and applied perspectives (pp. 1-27). Mahwah, NJ: Erlbaum.

Eschen, A., Freeman, J., Dietrich, T., Martin. M., Ellis, J., Martin, E., \& Kliegel, M. (2007). Motor brain regions are involved in the encoding of delayed intentions: A fMRI study. International Journal of Psychophysiology, 64, 259-268.

Forstmann, B. U., Brass, M., Koch, I., \& von Cramon, D. Y. (2005).
Internally generated and directly cued task sets: An investigation with fMRI. Neuropsychologia, 43, 943-952.

Forstmann, B. U., Wolfensteller, U., Derrfuss, J., Neumann, J., Brass, M., Ridderinkhof, K. R., \& von Cramon, D. Y. (2008). When the choice is ours: Context and agency modulate the neural bases of decision-making. PLOS ONE, 3(4), e1899.

Friston, K. J., Holmes, A. P., Worsley, K. J., Poline, J-P., Frith, C. D., \& Frackowiak, R. S. J. (1995). Statistical parametric maps in functional imaging: A general linear approach. Human Brain Mapping, 2, 189 210 .

Gilbert, S. J., Frith, C. D., \& Burgess, P. W. (2005). Involvement of rostral prefrontal cortex in selection between stimulus-oriented and stimulusindependent thought. European Journal of Neuroscience, 21, 14231431.

Gilbert, S. J., Simons, J. S., Frith, C. D., \& Burgess, P. W. (2006a). Performance-related activity in medial rostral prefrontal cortex (area 10) during low demand tasks. Journal of Experimental Psychology: Human Perception and Performance, 32, 45-58.

Gilbert, S. J., Spengler, S., Simons, J. S., Frith, C. D., \& Burgess, P. W (2006c). Differential functions of lateral and medial rostral prefrontal cortex (area 10) revealed by brain-behavior correlations. Cerebral Cortex, 16, 1783-1789.

Gilbert, S. J., Spengler, S., Simons, J. S., Steele, J. D., Lawrie, S. M., Frith, C. D., \& Burgess, P. W. (2006b). Functional specialization within rostral prefrontal cortex (area 10): A meta-analysis. Journal of Cognitive Neuroscience, $18,1-17$.

Gilbert, S. J., Williamson, I. D. M., Dumontheil, I., Simons, J. S., Frith, C. D., \& Burgess, P. W. (2007). Distinct regions of medial rostral prefrontal cortex supporting social and nonsocial functions. Social $\mathrm{Cog}$ nitive and Affective Neuroscience, 2, 217-226.

Gollwitzer, P. M. (1993). Goal achievement: The role of intentions. European Review of Social Psychology, 4, 141-185.

Gollwitzer, P. M. (1999). Implementation intentions: Strong effects of simple plans. American Psychologist, 54, 493-503.

Gollwitzer, P. M., \& Brandstätter, V. (1997). Implementation intentions and effective goal pursuit. Journal of Personality and Social Psychology, 73, 186-199.

Gollwitzer, P. M., \& Sheeran, P. (2006). Implementation intentions and goal achievement: A meta-analysis of effects and processes. Advances in Experimental Social Psychology, 38, 69-119.

Guynn, M. J. (2003). A two-process model of strategic monitoring in event-based prospective memory: Activation/retrieval mode and checking. International Journal of Psychology, 37, 245-256.

Hesse, M. D., Theil, C. M., Stephan, K. E., \& Fink, G. R. (2006). The left parietal cortex and motor intention: An event-related functional mag netic resonance imaging study. Neuroscience, 140, 1209-1221.

Kliegel, M., McDaniel, M. A., \& Einstein, G. O. (2008). Prospective memory: Cognitive, neuroscience, developmental, and applied perspectives. Mahwah, NJ: Erlbaum.

Kliegel., M., Martin, M., McDaniel, M. A., \& Einstein, G. O. (2001) Varying the importance of a prospective memory task: Differential effects across time- and event-based prospective memory. Memory, 9, $1-11$.

Koechlin, E., Basso, G., Pietrini, P., Panzer, S., \& Grafman, J. (1999, May 13). The role of anterior prefrontal cortex in human cognition. Nature, $399,148-151$

Lengfelder, A., \& Gollwitzer, P. M. (2001). Reflective and reflexive action control in frontal lobe patients. Neuropsychology, 15, 80-100.

Liu, L. L., \& Park, D. C. (2004). Aging and medical adherence: The use of automatic processes to achieve effortful things. Psychology and Aging, $19,318-325$

Marsh, R. L., Hicks, J. L., \& Cook, G. I. (2005). On the relationship between effort toward an ongoing task and cue detection in event-based 
prospective memory. Journal of Experimental Psychology: Learning, Memory, and Cognition, 31, 68-75.

McDaniel, M. A., \& Einstein, G. O. (2000). Strategic and automatic processes in prospective memory retrieval: A multiprocess framework. Applied Cognitive Psychology, 14, S127-\$144.

McDaniel, M. A., \& Howard, D. C. (2008). Implementation intentions facilitate prospective memory under high attention demands. Memory \& Cognition, 36, 716-724.

McNerney, M. W., \& West, R. (2007). An imperfect relationship between prospective memory and the prospective interference effect. Memory \& Cognition, 35, 275-282.

Monsell, S. (1996). Control of mental processes. In V. Bruce (Ed.), Unsolved mysteries of the mind (pp. 93-148). Hove, UK: Erlbaum/ Taylor \& Francis.

Nowinski, J. L., \& Dismukes, K. R. (2005). Effects of ongoing task context and target typicality on prospective memory performance: The importance of associative cueing. Memory, 13, 649-657.

Okuda, J., Fujii, T., Ohtake, H., Tsukiura, T., Yamadori, A., Frith, C. D., $\&$ Burgess, P. W. (2007). Differential involvement of regions of rostral prefrontal cortex (Brodmann area 10) in time- and event-based prospective memory. International Journal of Psychophysiology, 64, 233-246.

Okuda, J., Fujii, T., Yamadori, A., Kawashima, R., Tsukiura, T., Fukatsu, R., et al. (1998). Participation of the prefrontal cortices in prospective memory: Evidence from a PET study in humans. Neuroscience Letters, $253,127-130$.

Owen, A. M. (2006). The human ventrolateral frontal cortex and intended action. In N. Sebanz \& W. Prinz (Eds.), Disorders of volition (pp. 329-346). Cambridge, MA: MIT Press.

Owen, A. M., Herrod, N. J., Menon, D. K., Clark, J. C., Downey, S. P. M. J., Carpenter, A., et al. (1999). Redefining the functional organization of working memory processes within human lateral prefrontal cortex. European Joumal of Neuroscience, 11, 567-574.

Paul, I., Gawrilow, C., Zech, F., Gollwitzer, P., Rockstroh, B., Odenthal,
G., et al. (2007). If-then planning modulates the P300 in children with attention deficit hyperactivity disorder. Neuroreport, 18, 653-657.

Reynolds, J. R., West, R., \& Braver, T. (in press). Distinct neural circuits support transient and sustained processes in prospective memory and working memory. Cerebral Cortex.

Sakai, K., \& Passingham, R. E. (2003). Prefrontal interactions reflect future task operations. Nature Neuroscience, 6, 75-81.

Sakai, K., \& Passingham, R. E. (2006). Prefrontal set activity predicts rule-specific neural processing during subsequent cognitive performance. Journal of Neuroscience, 26, 1211-1218.

Simons, J. S., Schölvinck, M., Gilbert, S. J., Frith, C. D., \& Burgess, P. W. (2006). Differential components of prospective memory? Evidence from fMRI. Neuropsychologia, 44, 1388-1397.

Smith, R. E. (2003). The cost of remembering to remember in event-based prospective memory: Investigating the capacity demands of delayed intention performance. Journal of Experimental Psychology: Learning, Memory, and Cognition, 29, 347-361.

Waszak, F., Wenke, D., \& Brass, M. (2008). Cross-talk of instructed and applied arbitrary vasomotor mappings. Acta Psychologica, 127, 30-35.

West, R. (2008). The cognitive neuroscience of prospective memory. In M. Kliegel, M. A. McDaniel, \& G. O. Einstein (Eds.), Prospective memory. Cognitive, neuroscience, developmental, and applied perspectives (pp. 261-282). Mahwah, NJ: Erlbaum.

West, R., Krompinger, J., \& Bowry, R. (2005). Disruptions of preparatory attention contribute to failures of prospective memory. Psychonomic Bulletin and Review, 12, 502-507.

Wilson, E. A., \& Park, D. (2008). Prospective memory and health behaviors: Context trumps cognition. In M. Kliegel, M. A. McDaniel, \& G. O. Einstein (Eds.), Prospective memory: Cognitive, neuroscience, developmental, and applied perspectives (pp. 391-410). Mahwah, NJ: Erlbaum. 